\title{
Therapeutic Potential of Gut Microbiome Manipulation: Concepts in Fecal Microbiota Transplantation
}

\author{
Vinod Nikhra* \\ Department of Medicine, NDMC Medical College, India
}

Submission: February 06, 2019; Published: June 20, 2019

*Corresponding author: Vinod Nikhra, Senior Chief Medical Officer and Consultant, Department of Medicine, Hindu Rao Hospital and NDMC Medical College, New Delhi, India

\begin{abstract}
Microbial Alterations and Dysbiosis: The composition and diversity of gut microbiota is an indicator of health and various groups of commensal bacteria provide health advantages as they enhance metabolism, the immune system, cancer resistance, endocrine signaling and brain function. In general, the gut microbiome remains relatively resilient over time, however, antibiotic use, erratic diet, illness and other factors can lead to alterations and dysbiosis, which weaken various elements of the barrier, causing collapse of the mucus layer that separates epithelial cells and microbiota and reduced expression of antimicrobial peptides which control bacteria including C. difficile.
\end{abstract}

FMT- Invasive Gut Microbial Manipulation: FMT is administration of a form of fecal material from the donor into the intestinal tract of the recipient in order to directly modify the recipient's gut microbial composition suitably to confer health benefits. FMT has been used to successfully treat recurrent Clostridium difficile infection (DCI). There are preliminary indications to suggest that it may also carry therapeutic potential for other conditions such as inflammatory bowel disease, obesity, metabolic syndrome and functional gastrointestinal disorders.

Mechanisms and Effects of FMT: FMT involves administration of the whole microbiota from healthy donor stool into the recipient's intestinal tract to normalize or modify intestinal microbiota composition and function. FMT has restorative potential for both composition and functionality of gut microbiota and results in normalization of microbial diversity and community profile in patients by multiple mechanisms including competition for nutrients among C. difficile and other microbiota, direct suppression by antimicrobial peptides, bile-acid-mediated inhibition of spore germination and vegetative growth; and activation of immune-mediated colonization resistance.

Possible Scope of FMT Interventions: FMT is be considered for recurrent or relapsing CDI when there is failure to respond to conventional antibiotic therapy. For a moderate CDI, FMT is indicated when there is no response to standard therapy for at least 1 week. For severe CDI, it is indicated when there is no treatment response after appropriate maximal therapy for $48 \mathrm{~h}$. FMT leads to a significant change in microbial diversity in patients with recurrent CDI and complete resolution of symptoms. However, the trials to treat ulcerative colitis (UC) with FMT have shown conflicting results. Further, the patients with Crohn's disease (CD) and ulcerative colitis (UC) have increased incidence of CDI, and presence of CDI commonly complicates the course of these underlying diseases.

Limitations, Regulations and Complications: The occasional adverse effects of FMT are diarrhoea, abdominal cramping, belching and nausea, which are self-limiting and resolve in a variable period. An increased risk of IBD flare, fever and elevation in inflammatory markers following FMT may occur. Some serious adverse effects are upper GI bleeding, enteritis and peritonitis, which vary with the administration method and may be related to complications of the method itself rather than FMT. FMT is regulated in Canada as a 'new biologic drug', specifying the indications, patient's consent, preparing the FMT from a known solitary donor and screening for potential pathogens prior to administration. The US Food and Drug Administration (FDA) considers stool as a biological product and drug, and mandates physicians to maintain similar precautions to administer FMT. FMT, so far is not regulated in West by the European Medicines Agency, or elsewhere in Asia, Africa or Australia.

Future Directions and Developments: There is increasing acceptance for the therapeutic use of FMT. However, the range of risks and benefits remains poorly defined because the published FMT experience remains limited. In future, FMT can be a pauci-strain type or multistrain type depending on the fecal microbiota analysis of the recipient. The suitable strains can be picked-up from donor fecal sample, grown in cultures and transplanted through an appropriate route. Depending on the recipients' microbiota diagnostic analysis, the FMT using suitable pauci-strains may be a promising development in near future.

Keywords: Clostridium difficile infection; Fecal microbiota transplantation; Gut microbial diagnostics; IBD; IBS; MetS; Microbial Dysbiosis; Paucistrain and multi-strain transplantation; Pseudomembranous colitis

Abbreviations: DCI: Clostridium Difficile Infection; UC: Ulcerative Colitis; CD: Crohn’s Disease; FDA: Food and Drug Administration; PAMPs: Pathogen-Associated Molecular Patterns; NETs: Neutrophil Extracellular Traps; FMT: Faecal Microbiota Transplantation; DHA: Docosahexaenoic Acid; EPA: Eicosapentaenoic Acid; IBD: Inflammatory Bowel Disease; UC: Ulcerative Colitis; FIGD: Functional Gastrointestinal Disorders; IBS: Irritable Bowel Syndrome; IND: Investigational New Drug Application 


\section{Current Research in Diabetes \& Obesity Journal}

The Normal Gut Microbiome and Dysbiosis and Perturbed Alterations

The term 'microbiome' refers to the collective genomes of the microbiota that include organisms such as bacteria, archaea, microeukaryotes and viruses that reside in the human body and bear a commensal, symbiotic or pathogenic relationship. The human microbiota is estimated to contain 10-100 trillion microbial cells, of which the intestinal microbiota is the largest microbial population having over 1100 diverse species [1]. It has been estimated that the gut contains 1100 and at least 160 species per individual [2]. The human gut microbiota has been related to geography, race, ethnicity, and age and sex. The microbiota also varies in composition depending on the location along the gastrointestinal tract, like oesophageal, gastric, proximal intestinal, or distal intestinal) and axial depth (mucosal versus luminal). In addition, the diet also influences the gut microbiota composition [3].

It has been established that the composition and diversity of gut microbiota is an indicator of health [4]. The presence of various groups of commensal bacteria appear to provide health advantages. They have been shown to enhance metabolism, the immune system, cancer resistance, endocrine signaling and brain function. Some bacterial taxa associated with these benefits include Bacteroides, Bifidobacterium, Clostridium clusters XIVa/ IV and Lactobacillus. In general, the gut microbiome remains relatively resilient over time, however, antibiotic use, travel, erratic diet and illness can lead to its perturbed alterations [5].

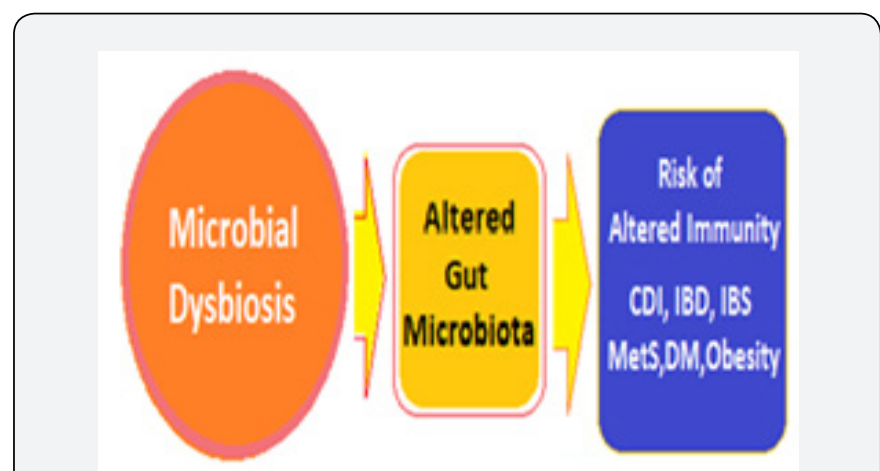

Figure 1: Microbial dysbiosis, altered microbiota and various fallouts.

Continuous signalling from indigenous microbiota maintains the optimal tone of gut barrier function and the mucosal immune system. In fact, there are multiple mucosal defences, which maintain compartmentalization of gut microbiota within the intestinal lumen, including the mucus layer, antimicrobial peptides, secreted immunoglobulins and a diverse cluster of mucosal lymphocytes. Further, the gut microbiome has well-defined ability to recover from potential insults. However, continued gastrointestinal and systemic stress can lead to a loss of this resilience and dysbiosis, and variable implications on health (Figure 1).

Intestinal infections and antibiotic treatments weaken various elements of the barrier, causing collapse of the mucus layer that separates epithelial cells and microbiota and reduced expression of antimicrobial peptides which targets Gram-positive bacteria including $C$. difficile. Once in contact with the intestinal epithelial layer, $C$. difficile toxins TcdA and TcdB inactivate small Rho-family regulatory GTPases by glycosylation and lead to disruption of essential cellular signalling pathways. Further, there occurs weakening of the tight junctions and apoptotic death of colonocytes, which results in opening of the epithelial barrier and interaction of toxins and $C$. difficile pathogen-associated molecular patterns (PAMPs) with resident mucosal immune cells.

The $C$. difficile toxins activate the inflammasome [6]. The massive neutrophil infiltration associated with CDI-induced pseudo-membranes results in rapid deployment of neutrophil extracellular traps (NETs) following release of granule proteins and chromatin. These NETs can patch the epithelial barrier defects and prevent gut leakage and microbial translocation. The need to repair the disrupted gut barrier and the exuberant immune response appears to be relevant in the context of acute pseudomembranous colitis.

\section{The Invasive Gut Microbial Manipulation}

\section{Basics of fecal microbiota transplantation}

The gut microbiota can be viewed as a microbial organ within the human body, which is made of communities of microorganisms representing the archaea, bacteria and eukarya, as well as viruses. These diverse microorganisms form intricate interactive and communicating networks made up by complex ecosystem and metabolic drivers. Thus, the gut microbiota has the potential to affect various physiological functions, including energy metabolism, immunity and neurological development and cognitive functions. In this respect, in addition to infectious risks, there are potential long-term risks for the recipient that should be considered in clinical practice of FMT.

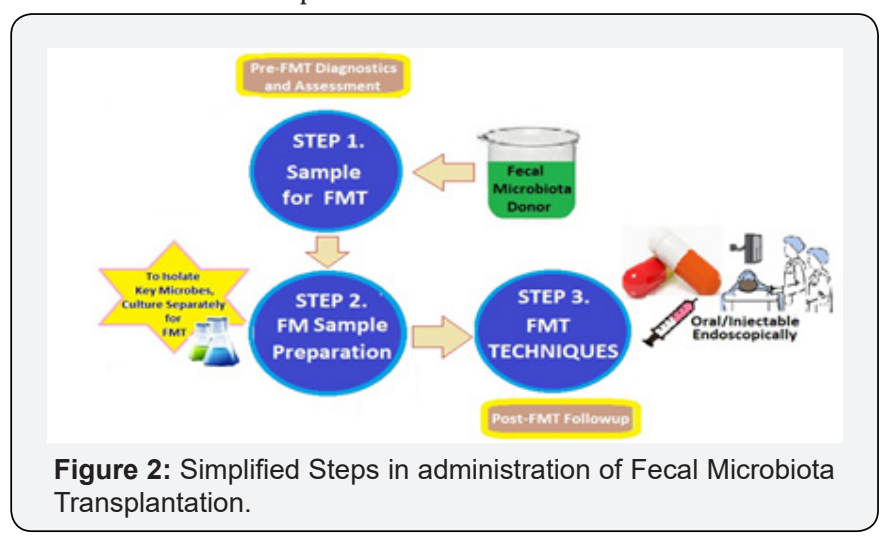

FMT is the administration of a form of fecal material from the donor into the intestinal tract of the recipient in order to directly modify the recipient's microbial composition suitably to confer health benefits [7]. First described by Eiseman and colleagues in 1958, the fecal enema was used as a treatment for pseudomembranous colitis [8]. Since then, FMT has been used to successfully treat recurrent Clostridium difficile infection (DCI). There are preliminary indications to suggest that it may also carry 


\section{Current Research in Diabetes \& Obesity Journal}

therapeutic potential for other conditions such as inflammatory bowel disease, obesity, metabolic syndrome and functional gastrointestinal disorders.

The FMT in modern medicine involves selecting a donor without a family history of autoimmune, metabolic and malignant diseases and screening for any potential pathogens. The material is then prepared by mixing fecal matter with water or normal saline, followed by filtration. The mixture is then administered through a nasogastric tube, esophago-gastroduodenoscopy, orally in capsule form, or through colonoscopy or retention enema (Figure 2). Most of the clinical experience with FMT has been derived from treating recurrent or refractory CDI.

\section{Alterations and Adaptations Following FMT}

FMT has the potential to restore the gut barrier by providing the necessary tonic signals for epithelial regeneration and production of mucins and anti-microbial peptides. There are required sequential multiple rounds of FMT, consistent with the time and impetus needed to repair the gut mucosa. Faecal microbiota transplantation (FMT) involves administration of the whole microbial Pool from healthy donor stool into the recipient's intestinal tract to normalize or modify intestinal microbiota composition and function. FMT has demonstrated its restorative potential for both composition and functionality of gut microbiota [9].

FMT results in normalization of microbial diversity and community structure in patients by multiple mechanisms including competition for nutrients among C. difficile and other microbiota, direct suppression by antimicrobial peptides, bile-acid-mediated inhibition of spore germination and vegetative growth; and activation of immune-mediated colonization resistance. Though, the mechanisms by which FMT exerts its therapeutic effects is not fully understood. Two broad, not mutually exclusive, mechanistic categories exist for the effectiveness of FMT that can be considered: The direct interaction of donor gut microbiota with $C$. difficile bacteria and microbiota mediated effects on host physiology and immune defences that are detrimental to $C$. difficile. Gut microbiota can compete with $C$. difficile for nutritional and colonization resources, interfere with its virulence factors and directly kill $C$. difficile bacteria. The gut micro biota can also activate multiple host immune defences and constrain $C$. difficile via secondary bile acids, which can be inhibitory of $C$. difficile germination and vegetative growth.

The most likely, there occurs a competitive exclusion of the pathogen, C. difficile, by the microbiota outcompeting for nutrients and creating an environment that is unfavourable for its growth [10]. Another factor is that the FMT restores the communities of Firmicutes and Bacteroidetes and decrease Proteobacteria favouring out-competing $C$. difficile. Further, the efficacy of FMT for rCDI through competitive exclusion occurs in part through the modulation of bile-salt metabolism, which affects $C$. difficile spore germination.
The primary bile acids stimulate germination of spores, whereas the secondary bile salts such as lithocholate serve as potent inhibitors for spore germination. Several groups of gut microbes found in healthy microbiota (primarily within the Lachnospiraceae and Ruminococcaceae families, also known as Clostridium clusters XIVa and IV) possess $7 \alpha$-dehydroxylation activity, which allows them to convert primary bile acids to secondary bile acids [11]. In accordance with this hypothesis, pre-FMT feces of rCDI patients were extremely low in secondary bile acids, but high in primary bile acids. After FMT, this trend was reversed, and resembled that of healthy stools from patients without CDI [12].

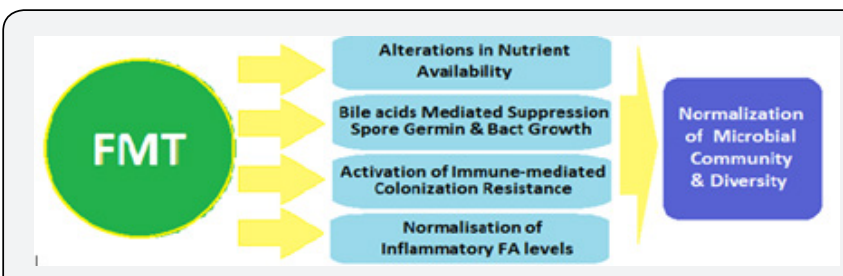

Figure 3: FMT: Mechanisms and Effects on the Gut Microbial Ecosystem.

Another important mechanism is that antibiotic treatment disrupts the endogenous microbial community and leads to increased free mucosal sialic acid (a carbohydrate energy source for $C$. difficile), which leads to expansion of $C$. difficile colonies in the gut [13] The FMT appears to increase sialic-acid utilization by commensal bacteria, thus depriving $C$. difficile of the vital energy source. Yet, other likely mechanisms may be the enhanced post-FTM protease activity inactivating secreted $C$. difficile toxins, stimulation of host-cell defence through release of small molecules such as short-chain fatty acids and a direct activity against $C$. difficile viability through bacteriocin-like mechanisms [14]. The FMT moves the microbiota of recipients towards that of the donor and improves bacterial diversity. There occur changes in community structure and composition in the microbiota at phylum and genus levels after FMT. Faecal bile acid levels which are significantly different in donors and recipients, turn more donor-like in FMT recipients (Figure 3).

Inflammatory mediators are the link between innate and adaptive immunity. Inappropriate damage to host tissue through uncontrolled inflammation via pathogen toxins or endotoxins appear to release cellular fatty acids in CD infection. Fatty acids, particularly PUFA metabolised to eicosanoids, are a key factor to mediate and regulate the inflammation response. $\omega-3$ PUFA and arachidonic acid is a key precursor of inflammatory cells and its levels dictate the duration and intensity of the response.

Whereas, $\omega-6$ PUFA Docosahexaenoic acid (DHA) and Eicosapentaenoic acid (EPA) moderate the effect of arachidonic acid and its downstream products through anti-inflammatory resolvins, docosatrienes, neuro-protectins for DHA and E series resolvins via EPA. There is a proposed link between PUFAs, gut health and elevated levels of fatty acids found in patient groups 


\section{Current Research in Diabetes \& Obesity Journal}

relative to donors. This includes SCFA, pyruvic acid and lactic acid, saturated fatty acids palmitic acid, and myristic acid - also an $\omega-6$ FA, monounsaturated FAs, oleic acid and palmitoleic acid, $\omega$-3 PUFAs $\alpha$-linolenic acid and $\gamma$-linolenic acid (ALA+GLA) and EHA and DHA as well as, $\omega-6$ Linoleic acid and arachidonic acid. There occurs normalisation of individual inflammatory FA levels in recipients. SCFA, $\omega-3 \& \omega-6$ PUFAs are altered in the direction of the donor following FMT [15].

FMT leads to a significant change in microbial diversity in patients with recurrent CDI and complete resolution of symptoms. Stool donor type (related or unrelated) and degree of engraftment are not the key for successful treatment of CDI by FMT. However, CDI patients with IBD have higher proportion of the original community after FMT and lack of improvement of their IBD symptoms and increased episodes of CDI on long-term follow-up [16].

\section{Possible scope of FMT interventions}

\section{Clostridium difficile infection}

Normally, a diverse gut microbial community confers colonization resistance against pathogens such as C. difficile. But, the disruptions due to antibiotics, comorbidities, altered gastrointestinal transit, or other risk factors predispose to pathogen colonization and infection [17]. The deleterious shift in the gut microbial ecosystem, referred to as dysbiosis, is associated with Clostridium difficile infection (CDI), inflammatory bowel disease (IBD) and other systemic inflammatory and metabolic conditions [18]. There is an increasing incidence of community and hospital acquired CDI and high rates of recurrent CDI. The stress on newer non-antibiotic therapies has led to the emergence of microbiome-based therapies [19]. FMT in CDI patients has been shown to restore the phylogenetic diversity by enema, oral capsule, or endoscopic delivery modes [20,21].

The CDI patients are routinely treated with metronidazole or vancomycin required for a significant number of patients. Some patients with CDI go on to develop recurrent CDI (rCDI), having significant higher morbidity and mortality. Antibiotics have been implicated in the pathogenesis of CDI, as the antibiotic use can lead to dysbiosis allowing $C$. difficile to flourish. The use of fidaxomicin has lower recurrence rates due to a narrower spectrum of antimicrobial activity and thus limiting dysbiosis. The use of FMT for rCDI has an average of $87-90 \%$ cure rate with resolution of diarrhoea. Moreover, FMT leads to normalization and restoration of gut microbiota [22]. A randomized control trial has shown that duodenal infusion of donor feces for rCDI had a cure rate of $81 \%$ versus a cure rate of $31 \%$ for patients treated with the standard course of oral vancomycin [23].

FMT should be considered for recurrent or relapsing CDI when there is failure to respond to conventional antibiotic therapy. For a moderate CDI, FMT is indicated when there is no response to standard therapy for at least 1 week. For severe CDI, it is indicated when there is no treatment response after appropriate maximal therapy for $48 \mathrm{~h}$. FMT is also supported by the 2013 American
College of Gastroenterology C. difficile-treatment guidelines as a therapeutic alternative for rCDI that has not responded to a pulse/ tapered regimen of vancomycin [24]. The use of frozen donor feces administered by colonoscopy, which has also been shown to be effective [25]. In a recent study there was documented significantly lower response of CDI to FMT in patients with underlying IBD [26]. Also, the rate of recurrence of CDI following FMT is high in patients with CDI and underlying IBD [27].

The human gut microbiota is a diverse ecosystem consisting of thousands of bacterial species, protects against invasive pathogens such as C. difficile, which is a Gram-positive, anaerobic, spore-forming, and toxin-producing bacillus. C. difficile infection is a model for disease caused by the host gut-microbial dysbiosis. The CDI causes symptoms ranging from mild watery diarrhoea to potentially lethal pseudomembranous colitis. The pathogenesis of CDI requires disruption of the gut microbiota before onset of symptomatic disease, and exposure to antibiotics is the most common precipitant.

Following exposure, the manifestations can vary from asymptomatic colonization, to a self-limited diarrheal illness, to a fulminant, life-threatening colitis. Even among those that recover, recurrent disease is common. Symptomatic Clostridium difficile infection (CDI) results when $C$. difficile, a Gram-positive bacillus that is an obligate anaerobe, produces cytotoxins TcdA and TcdB, causing epithelial and mucosal injury in the gastrointestinal tract $[28,29]$.

The altered intestinal microbiota is a prerequisite to symptomatic infection. Following establishment of susceptibility and exposure to spores, germination occurs, vegetative $C$. difficile cells produce toxin, and this causes injury to the intestinal epithelium and mucosa resulting in symptoms. After recovery following conventional therapy, the disruption of the intestinal microbiota may continue, and patients remain at risk for rCDI [30]. Conventional treatments utilize antibiotics with activity against $C$. difficile, but these antibiotics have activity against other gut bacteria, limiting the ability of the microbiota to fully recover following CDI and predisposing patients to recurrence.

Traditional treatments for CDI result in a high incidence of recurrence (35\%), with up to $65 \%$ of these patients that are again treated with conventional approaches developing a chronic pattern of recurrent CDI. fidaxomicin is considerably more expensive than vancomycin, is approved by the FDA for the treatment of CDI. The restoration of the gut microbiome through fecal microbiota transplantation (FMT) is the strategic treatment that has gained acceptance for the treatment of recurrent CDI when conventional treatments have failed [31].

Evidence for Efficacy of FMT in Treatment of CDI: The clinical evidence for FMT is most robust for recurrent CDI, consisting of case reports or case series, recently aggregated by two large systematic reviews, as well as several clinical trials [32,33]. Further, FMT for treatment of recurrent CDI has remarkable success rates for alleviation of the symptoms and restoration of 


\section{Current Research in Diabetes \& Obesity Journal}

health. The effectivity of FMT in restoring a microbiome in the GIT, appears to be brought about by the long-term successful and stable reconstruction of commensal microbes, which access and occupy the niches in the GI space following transplantation [34].

\section{Inflammatory bowel disease}

IBD is an intestinal disorder that includes ulcerative colitis (UC) and Crohn's disease (CD). IBD is characterized by chronic inflammation of the gastrointestinal tract, having a cyclic nature of disease progression and remission. During periods of disease activity, patients may present with diarrhoea, nausea, weight loss, loss of appetite, fever, and abdominal pain. The pathophysiology is multifactorial, due to imbalances in the intestinal microbiota, gut epithelium, and immune system in genetically susceptible individuals. IBD is hypothesized to occur due to continuous inappropriate antigenic stimulation of gut mucosa-associated lymphatic tissue by commensal microbes [35]. Dysbiosis of the gut is considered as a possible pathologic contributor to IBD development. This idea is supported by observations that antibiotics such as amoxicillin/clavulanic acid and rifaximin can reduce intestinal inflammation and induce remission in some patients [36].

Metagenomic and metabolomics studies have characterized the IBD microbiota, and have found an overall reduced bacterial diversity, with specifically reduced members of the Bacteroidetes phylum and the Lachnospiraceae group within the Firmicutes phylum and an increase in Proteobacteria and Actinobacteria [37]. Biopsy specimens from patients with $C D$ were found to have a reduced population of the Clostridium cluster IV species, Faecalibacterium prausnitzii. F. prausnitzii is associated with anti-inflammatory properties in patients with CD, and increased levels of the bacterium are associated with maintenance of clinical remission in UC [38,39].

Overall, the IBD microbiome was found to be inflammation promoting, with indications of increased oxidative stress, increased type II toxin secretion, and increased virulence-related bacterial genes [40]. It has been shown that the transplantation of fecal ecosystems from patients with UC to germ-free mice increased sensitivity to dextran sodium sulfate-induced colitis. Thus, supporting the use of microbiota modification for the treatment of UC [41]. The evidence that links gut microbial dysbiosis with IBD has led to the exploration of FMT as therapy for the disease [42].

It appears that FMT may be more effective for $\mathrm{CD}$ and in younger patients than for UC infection. In the trial by Moayyedi and colleagues, the patients that benefitted most from FMT were those with a recent history of disease onset [43]. This may indicate that FMT may be useful only in certain subsets of patients with UC. Although no serious adverse events are in the IBD patients treated with FMT, some side effects include fevers, chills, bloating, flatulence, vomiting, diarrhoea and abdominal tenderness [44]. Also, some patients' conditions may worsen after FMT. However, it is apparent that FMT is not as effective in IBD as it is in CDI, which is probably due to the multifactorial pathophysiology of IBD [45].

\section{Functional gastrointestinal disorders (FIGD)}

Currently, FGID the most common GI disease. It is characterized by the presence of GI symptoms in the absence of any identifiable anatomic or biochemical abnormalities. Irritable bowel syndrome (IBS) is the most prevalent form of FGID, affecting about 10-15\% of the population and having impact on the QOL. IBS has four subtypes based on the dominant symptoms: IBS-D is diarrhoeapredominant, IBS-C is constipation predominant, IBS-M is mixed diarrhoea and constipation, and IBS-U is an un-subtyped [46].

The pathophysiology involves visceral hypersensitivity, altered barrier function, altered GI motility, and an altered gutbrain axis. These changes have been related to alterations in the gut microbiota [47]. The therapeutic beneficial effect of FMT has been shown in varying proportion of limited number of IBS patients. In a study, FMT was administered to 13 patients with IBS ( 9 with IBS-D, 3 with IBS-C, 1 with IBS-M) via esophagogastroduodenoscopy, documented symptomatic relief in about $70 \%$ patients for a variable period of 6-18 months [48]. The data for FTM treatment of FGID are limited and may be biased.

\section{Obesity and metabolic syndrome}

Various studies indicate that the gut microbiota may be involved in the pathophysiology of obesity [49]. The metagenomic studies have documented that the gut microbiome in lean and obese individuals has marked differences. The obese gut microbiota of the mice studied showed an increase in the Firmicutes to Bacteroidetes ratio and had an increased capacity for energy extraction from dietary intake.

Moreover, Turnbaugh and colleagues showed that the colonization of germ-free mice with the obese microbiota resulted in a significantly greater increase in adiposity than those transplanted with the lean microbiota [50]. Obese individuals have been found to have a relative abundance of genes involved in hydrogen and methane production, and a relative decrease in genes associated with hydrogen sulphide production [51]. Transfer of the gut microbiota from human twins discordant for obesity into germ-free mice led to greater adiposity and body mass in the mice transplanted with the obese microbiota.

The altered microbiota found in obese individuals may be predisposing them to obesity through increased energy extraction, or possibly through an interaction with the gut-brain access leading to decreased energy output or through influencing satiety. The fecal transplants from lean to obese (with metabolic syndrome) individuals resulted in improved insulin sensitivity, increased gut-microbial diversity, and increased butyrateproducing bacteria (Roseburia intestinalis) in the obese recipients [52]. Conversely, the potential of the gut microbiota to affect weight gain has led to the proposal that the body mass index of the donor may need to be taken into consideration when choosing candidate donors for FMT [53]. 


\section{Current Research in Diabetes \& Obesity Journal}

\section{The FMT: Limitations and Complications}

The clinical experience with FMT has mostly come from its use in treating rCDI. The FMT has been found to be relatively free of adverse effects [54]. The adverse effects are diarrhoea, abdominal cramping, belching and nausea, and are often self-limiting and resolved in a variable period [55]. Aside from the minor selflimiting adverse effects, some serious adverse documented are upper GI bleeding, enteritis and peritonitis [56]. Further, as longterm adverse effects of FMT, there exists a theoretical possibility of unrecognized infectious disease transfer or stimulation of chronic disease related to alteration of the gut microbiota like obesity, diabetes, atherosclerosis, etc. The advances in FMT delivery are likely to reduce adverse effects in the future.

Short-term complications: Serious adverse effects directly attributable to FMT in patients with normal immune function are uncommon. Symptoms of an irritable bowel (constipation, diarrhoea, cramping, bloating) shortly after FMT are observed and usually last less than 48 hours [57]. An increased risk of IBD flare, fever, and elevation in inflammatory markers following FMT has also been observed. FMT side effects may vary by the administration method and may be related to complications of the method itself rather than FMT.

Long-term complications: The long-term safety of FMT is unknown [54]. There is an incomplete understanding of the interaction between the gut microbiome and the host, but this is a complex system and associations with disease processes have been demonstrated. The gut microbiome may be associated with colon cancer, diabetes, obesity, and atopic disorders. The role of FMT in contributing to these conditions is unknown. Based on several prospective trials and observational data, FMT appears to be a safe and effective treatment for recurrent CDI that is superior to conventional approaches.

An important subpopulation of patients with rCDI has underlying IBD which contributes to gut dysbiosis. The trials to treat ulcerative colitis (UC) with FMT have shown conflicting results [58-60] Further, the patients with Crohn's disease (CD) and ulcerative colitis (UC) have increased incidence of CDI, and presence of CDI commonly complicates the course of these underlying diseases [61] The FMT has not shown consistent success in treating diseases like IBD. The underlying host factors like gut microbial ecology may be important for success pre- and post-FMT.

\section{The Ethical Issues and Regulations}

Health Canada released an interim policy regarding the regulation of FMT, which it currently regulates as a 'new biologic drug'. It specifies the indications, patient's consent, preparing the FMT from a known solitary donor and screened for potential pathogens prior to administration. This policy limits the use of FMT for other conditions apart from rCDI, due to the paucity of data from large clinical trials. Similarly, the US Food and Drug Administration (FDA) considers stool as a biological product and drug, and mandates physicians to obtain an investigational new drug application (IND) to administer FMT.

Recently, the FDA has stated that it will allow physicians to use their own discretion in administering FMT to patients with CDI that does not respond to conventional therapies (without an IND). The current Food and Drug Administration guidance allows performance of FMTs in treatment of CDI failing standard antibiotic therapies but requires provision of an informed consent, explaining the risks and benefits of the procedure, and explain that it is an investigational therapy. Also, the donor must be from the similar social and geographical community, known to the patient or healthcare provider, and the stool must be screened for pathogens [62]. But, FMT, so far is not regulated in West by the European Medicines Agency, or elsewhere within China or Australia.

\section{Future Directions and Developments}

There is increasing acceptance for the therapeutic use of FMT, partially due to its perception as a 'natural' treatment and its relatively inexpensive implementation. However, the range of risks and benefits remains poorly defined because the published FMT experience remains limited.

There are fears of the infectious potential of the therapy, which have led researchers to explore the use of 'synthetic stool' products with defined bacterial populations to ameliorate such concerns [63]. There are many efforts currently underway to explore a role for the gut microbiota in the pathophysiology of many other conditions, including necrotizing enterocolitis, liver disease, colorectal cancer, oesophageal and gastric adenocarcinoma and autism $[64,65]$. In addition, there is a possible role for gutmicrobiota modification in many other conditions [66]. Depending on the research in this field, FMT may be a potential therapy for additional conditions in the future.

Several host factors likely play significant roles in the pathophysiology of rCDI and can affect risks and benefits of FMT and clinical care of patients after the procedure. As more options for FMT emerge, patients will likely opt for less invasive mechanisms, such as oral delivery capsules [67]. However, underlying gastrointestinal pathology should be considered in patients with refractory RCDI and colonoscopic evaluation may provide valuable diagnostic information [68-70].

In future, FMT can be a pauci-strain type or multi-strain type depending on the fecal microbiota analysis of the recipient. The suitable strains can be picked-up from donor fecal sample, grown in cultures and transplanted through an appropriate route. Depending on the recipients' microbiota diagnostic analysis, the FMT using suitable pauci-strains may be a promising development.

\section{References}

1. Hongoh Y (2010) Diversity and genomes of uncultured microbial symbionts in the termite gut. Biosci Biotechnol Biochem 74(6): 1145-1151.

2. Qin J, Li R, Raes J, Arumugam M, Burgdorf KS, et al. (2010) A human 


\section{Current Research in Diabetes \& Obesity Journal}

gut microbial gene catalogue established by metagenomic sequencing Nature 464(7285): 59-65.

3. David LA, Maurice CF, Carmody RN, Gootenberg DB, Button JE, et al. (2014) Diet rapidly and reproducibly alters the human gut microbiome. Nature 505(7484): 559-563.

4. Claesson MJ, Jeffery IB, Conde S, Power SE, O'Connor EM, et al. (2012) Gut microbiota composition correlates with diet and health in the elderly. Nature 488(7410): 178-184.

5. Malla MA, Dubey A, Kumar A, Yadav S, Hashem A, et al. (2019) Exploring the Human Microbiome the Potential Future Role of Next-Generation Sequencing in Disease Diagnosis and Treatment. Front Immunol 9: 2868 .

6. Khoruts A, Sadowsky MJ (2016) Understanding the mechanisms of faecal microbiota transplantation. Nat Rev Gastroenterol Hepatol 13(9): 508-516.

7. Gupta S, Allen-Vercoe E, Petrof EO (2016) Fecal microbiota transplantation in perspective. Therap Adv Gastroenterol 9(2): 229-239.

8. Eiseman B, Silen W, Bascom GS, Kauvar AJ (1958) Fecal enema as an adjunct in the treatment of pseudomembranous enterocolitis. Surgery 44(5): 854-859

9. Smits LP, Bouter KE, de Vos WM, Borody TJ, Nieuwdorp M (2013) Therapeutic potential of fecal microbiota transplantation. Gastroenterology 145(5): 946-953.

10. Kelly C, Kahn S, Kashyap P, Laine L, Rubin D, et al. (2015) Update on fecal microbiota transplantation 2015: indications, methodologies, mechanisms, and outlook. Gastroenterology 149(1): 223-227.

11. Sorg J, Sonenshein A (2008) Bile salts and glycine as cogerminants for Clostridium difficile spores. J Bacteriol 190(7): 2505-2512.

12. Weingarden A, Chen C, Bobr A, Yao D, Lu Y, et al. (2014) Microbiota transplantation restores normal fecal bile acid composition in recurrent Clostridium difficile infection. Am J Physiol Gastrointest Liver Physiol 306(4): G310-G319.

13. Ng K, Ferreyra J, Higginbottom S, Lynch JB, Kashyap PC, et al. (2013) Microbiota-liberated host sugars facilitate post-antibiotic expansion of enteric pathogens. Nature 502(7469): 96-99.

14. Rea M, Sit C, Clayton E, O’Connor PM, Whittal RM, et al. (2010) Thuri cin $\mathrm{CD}$, a posttranslationally modified bacteriocin with a narrow spectrum of activity against Clostridium difficile. Proc Natl Acad Sci U S A 107(20): 9352-9357.

15. Brown JR, Flemer B, Joyce SA, Zulquernain A, Sheehan D, et al. (2018) Changes in microbiota composition bile and fatty acid metabolism in successful faecal microbiota transplantation for Clostridioides difficile infection. BMC Gastroenterol 18(1): 131.

16. Khanna S, Vazquez-Baeza Y, González A, Weiss S, Schmidt B, et al. (2017) Changes in microbial ecology after fecal microbiota transplantation for recurrent $C$. difficile infection affected by underlying inflammatory bowel disease. Microbiome 5(1): 55.

17. Khanna S, Pardi DS (2016) Clinical implications of antibiotic impact on gastrointestinal microbiota and Clostridium difficile infection. Expert Rev Gastroenterol Hepatol 10(10): 1145-1152.

18. Khanna S, Tosh PK (2014) A clinician's primer on the role of the microbiome in human health and disease. Mayo Clin Proc 89(1): 107-114.

19. Lessa FC, Winston LG, McDonald LC (2015) Emerging Infections Program CdST Burden of Clostridium difficile infection in the United States. N Engl J Med 372(24): 2369-2370.

20. Orenstein R, Dubberke E, Ray A, Mullane K, Hardi R, et al. (2016) Safety and durability of RBX2660 (microbiota suspension) for recurrent Clostridium difficile infection results of the PUNCH CD study. Clin Infect Dis 62(5): 596-602.
21. Khanna S, Pardi DS, Kelly CR, Kraft CS, Dhere T, et al. (2016) A novel microbiome therapeutic increases gut microbial diversity and prevents recurrent Clostridium difficile infection. J Infect Dis 214(2): 173-181.

22. Rossen NG, MacDonald JK, de Vries EM, D’Haens GR, de Vos WM, et al. (2015) Fecal microbiota transplantation as novel therapy in gastroenterology: A systematic review. World J Gastroenterol 21(17): 53595371.

23. van Nood E, Vrieze A, Nieuwdorp M, Zoetendal EG, de Vos WM, et al. (2013) Duodenal infusion of donor feces for recurrent Clostridium difficile. N Engl J Med 368(5): 407-415.

24. Surawicz CM, Brandt LJ, Binion DG, Ananthakrishnan AN, Curry SR, et al. (2013) Guidelines for diagnosis, treatment, and prevention of Clostridium difficile infections. Am J Gastroenterol 108(4): 478-498.

25. Hamilton MJ, Weingarden AR, Sadowsky MJ, Khoruts A (2012) Standardized Frozen Preparation for Transplantation of Fecal Microbiota for Recurrent Clostridium difficile Infection. Am J of Gastroenterology 107(5):761-767.

26. Khoruts A, Rank KM, Newman KM, Viskocil K, Vaughn BP, et al. (2016) Inflammatory bowel disease affects the outcome of fecal microbiota transplantation for recurrent Clostridium difficile infection. Clin Gastroenterol Hepatol 14(10): 1433-1438.

27. Khanna S, Weatherly R, Kammer PP, Pardi DS (2015) Management and outcomes of patients with failed fecal microbiota transplantation for recurrent Clostridium difficile infection. Am J Gastroenterol 14(10): S580.

28. Lessa FC, Mu Y, Bamberg WM, Beldavs ZG, Dumyati GK, et al. (2015) Burden of Clostridium difficile infection in the United States. N Engl J Med 372(9): 825-834

29. He M, Miyajima F, Roberts P, Ellison L, Pickard DJ, et al. (2013) Emergence and global spread of epidemic healthcare-associated Clostridium difficile. Nat Genet 45(1): 109-113.

30. Abou Chakra CN, Pepin J, Sirard S, Valiquette L (2014) Risk Factors for Recurrence, Complications and Mortality in Clostridium difficile Infection: A Systematic Review. PLoS ONE 9(6): e98400.

31. Rao K, Safdar N (2016) Fecal Microbiota Transplantation for the Treatment of Clostridium difficile Infection. J Hosp Med 11(1): 56-61.

32. Kassam Z, Lee CH, Yuan Y, Hunt RH (2013) Fecal Microbiota Transplantation for Clostridium difficile Infection: Systematic Review and Meta-Analysis. Am J Gastroenterol 108(4): 500-508.

33. Kumar R, Maynard CL, Eipers P, Goldsmith KT, Ptacek T, et al. (2016). Colonization potential to reconstitute a microbe community in patients detected early after fecal microbe transplant for recurrent $C$. difficile. BMC Microbiol 16: 5 .

34. Landy J, Al-Hassi HO, McLaughlin SD, Walker AW, Ciclitira PJ, et al. (2011) Review article: faecal transplantation therapy for gastrointestinal disease. Aliment Pharmacol Ther 34(4): 409-415.

35. Zhang Y, Li Y (2014) Inflammatory bowel disease: pathogenesis. World J Gastroenterol 20(1): 91-99.

36. Khan K, Ullman T, Ford A, Abreu MT, Abadir A, et al. (2011) Antibiotic therapy in inflammatory bowel disease: a systematic review and meta-analysis. Am J Gastroenterol 106(4): 661-673.

37. Morgan X, Tickle T, Sokol H, Gevers D, Devaney KL, et al. (2012) Dysfunction of the intestinal microbiome in inflammatory bowel disease and treatment. Genome Biol 13(9): R79.

38. Willing B, Halfvarson J, Dicksved J (2009) Twin studies reveal specific imbalances in the mucosa-associated microbiota of patients with ileal Crohn's disease. Inflamm Bowel Dis 15: 653-660.

39. Varela E, Manichanh C, Gallart M, Torrejón A, Borruel N, et al. (2013) Colonisation by Faecalibacterium prausnitzii and maintenance of clin- 


\section{Current Research in Diabetes \& Obesity Journal}

ical remission in patients with ulcerative colitis. Aliment Pharmacol Ther 38(2): 151-161.

40. Erickson A, Cantarel B, Lamendella R, Darzi Y, Mongodin EF, et al. (2012) Integrated metagenomics/metaproteomics reveals human host-microbiota signatures of Crohn's disease. PLoS One 7(1): e49138.

41. Natividad J, Pinto-Sanchez M, Galipeau H, Jury J, Jordana M, et al (2015) Ecobiotherapy rich in firmicutes decreases susceptibility to colitis in a humanized gnotobiotic mouse model. Inflamm Bowel Dis 21(8): 1883-1893.

42. Damman C, Miller S, Surawicz C, Zisman TL (2012) The microbiome and inflammatory bowel disease: Is there a therapeutic role for fecal microbiota transplantation? Am J Gastroenterol 107: 1452- 1059.

43. Moayyedi P, Surette M, Kim P, Libertucci J, Wolfe M, et al. (2015) Fecal microbiota transplantation induces remission in patients with active ulcerative colitis in a randomized, controlled trial. Gastroenterology 149(1): 102-109.

44. Suskind D, Brittnacher M, Wahbeh G, Shaffer ML, Hayden HS, et al (2015) Fecal microbial transplant effect on clinical outcomes and fecal microbiome in active Crohn's disease. Inflamm Bowel Dis 21(3) 556-563.

45. De Leon L, Watson J, Kelly C (2013) Transient flare of ulcerative colitis after fecal microbiota transplantation for recurrent Clostridium difficile infection. Clin Gastroenterol Hepatol 11(8): 1036-1038.

46. Yao X, Yang Y, Cui L, Zhao KB, Zhang ZH, et al. (2012) Subtypes of irritable bowel syndrome on Rome III criteria: a multicenter study. J Gastroenterol Hepatol 27(4): 760-765.

47. Pinn D, Aroniadis O, Brandt L (2015) Is fecal microbiota transplantation (FMT) an effective treatment for patients with functional gastrointestinal disorders (FGID)? Neurogastroenterol Motil 27(1): 19-29.

48. Pinn D, Aroniadis O, Brandt L (2013) Follow-up study of fecal microbiota transplantation (FMT) for the treatment of refractory irritable bowel syndrome (IBS). Presented at the American College of Gastroenterology Annual Meeting; San Diego, CA, USA.

49. Liou A, Paziuk M, Luevano J, Machineni S, Turnbaugh PJ, et al. (2013) Conserved shifts in the gut microbiota due to gastric bypass reduce host weight and adiposity. Sci Transl Med 5(178): 178ra141.

50. Turnbaugh P, Ley R, Mahowald M, Magrini V, Mardis ER, et al. (2006) An obesity-associated gut microbiome with increased capacity for energy harvest. Nature 444(7122): 1027-1031.

51. Le Chatelier E, Nielsen T, Qin J, Prifti E, Hildebrand F, et al. (2013) Richness of human gut microbiome correlates with metabolic markers. Nature 500(7464): 541-546.

52. Vrieze A, Van Nood E, Holleman F, Salojärvi J, Kootte RS, et al. (2012) Transfer of intestinal microbiota from lean donors increases insulin sensitivity in individuals with metabolic syndrome. Gastroenterology 143(4): 913-916.

53. Alang N, Kelly C (2015) Weight gain after fecal microbiota transplantation. Open Forum Infect Dis 2(1): 4.

54. Brandt L, Aroniadis O, Mellow M, Kanatzar A, Kelly C, et al. (2012) Long-term follow-up of colonoscopic fecal microbiota transplant for recurrent Clostridium difficile infection. Am J Gastroenterol 107(7): 1079-1087.

55. Van Nood E, Speelman P, Nieuwdorp M, Keller J (2014) Fecal microbiota transplantation: facts and controversies. Curr Opin Gastroenterol 30(1): 34-39.
56. Kassam Z, Lee C, Yuan Y, Hunt R (2013) Fecal microbiota transplantation for Clostridium difficile infection: systematic review and meta-analysis. Am J Gastroenterol 108(4): 500-508.

57. Gough E, Shaikh H, Manges AR (2011) Systematic Review of Intestinal Microbiota Transplantation (Fecal Bacteriotherapy) for Recurrent Clostridium difficile Infection. Clin Infect Dis 53(10): 994-1002.

58. Llopis M, Cassard-Doulcier A, Boschat L (2014) Intestinal dysbiosis explains inter-individual differences in susceptibility to alcoholic liver disease. J Hepatol 60(Suppl): S1-S598.

59. Borody T, Khoruts A (2012) Fecal microbiota transplantation and emerging applications. Nat Rev Gastroenterol Hepatol 9(2): 88-96.

60. Moayyedi P, Surette MG, Kim PT, Libertucci J, Wolfe M, et al. (2015) Fecal microbiota transplantation induces remission in patients with active ulcerative colitis in a randomized controlled trial. Gastroenterology 149(1): 102-109.

61. Rossen NG, Fuentes S, van der Spek MJ, Tijssen JG, Hartman JH, et al. (2015) Findings from a randomized controlled trial of fecal transplantation for patients with ulcerative colitis. Gastroenterology 149(1): 110-118.

62. (2014) Draft Guidance for Industry: Enforcement Policy Regarding Investigational New Drug Requirements for Use of Fecal Microbiota for Transplantation to Treat Clostridium difficile Infection Not Responsive to Standard Therapies. Silver Spring, MD: US Food and Drug Administration.

63. Petrof E, Gloor G, Vanner S, Weese SJ, Carter D, et al. (2013) Stool substitute transplant therapy for the eradication of Clostridium difficile infection: 'repopulating' the gut. Microbiome 1(1): 3.

64. Buie T (2015) Potential etiologic factors of microbiome disruption in autism. Clin Ther 37(5): 976-983.

65. Paramsothy S, Kamm MA, Kaakoush NO, Walsh AJ, van den Bogaerde J, et al. (2017) Multidonor intensive faecal microbiota transplantation for active ulcerative colitis: a randomised placebo-controlled trial. Lancet 389(10075): 1218-1228.

66. Khanna S, Vazquez-Baeza Y, González A, Weiss S, Schmidt B, et al. (2017) Changes in microbial ecology after fecal microbiota transplantation for recurrent $C$. difficile infection affected by underlying inflammatory bowel disease. Microbiome 5(1): 55.

67. Khoruts A, Rank KM, Newman KM, Viskocil K, Vaughn BP, et al. (2016) Inflammatory Bowel Disease Affects the Outcome of Fecal Microbiota Transplantation for Recurrent Clostridium difficile Infection. Clin Gastroenterol Hepatol 14(10):1433-1438.

68. Kostic AD, Xavier RJ, Gevers D (2014) The microbiome in inflammatory bowel disease: current status and the future ahead. Gastroenterology 146: 1489-1499.

69. Ananthakrishnan AN (2012) Detecting and treating Clostridium difficile infections in patients with inflammatory bowel disease. Gastroenterol Clin North Am 41(2): 339-353.

70. Agrawal M, Aroniadis OC, Brandt LJ, Kelly C, Freeman S, et al. (2016) The long-term efficacy and safety of fecal microbiota transplant for recurrent, severe, and complicated Clostridium difficile infection in 146 elderly individuals. J Clin Gastroenterol 50(5): 403-407. 
(C) This work is licensed under Creative

CC) (1) Commons Attribution 4.0 Licens

BY DOI: 10.19080/CRDOJ.2019.11.555803
Your next submission with Juniper Publishers will reach you the below assets

- Quality Editorial service

- Swift Peer Review

- Reprints availability

- E-prints Service

- Manuscript Podcast for convenient understanding

- Global attainment for your research

- Manuscript accessibility in different formats

( Pdf, E-pub, Full Text, Audio)

- Unceasing customer service

Track the below URL for one-step submission

https://juniperpublishers.com/online-submission.php 